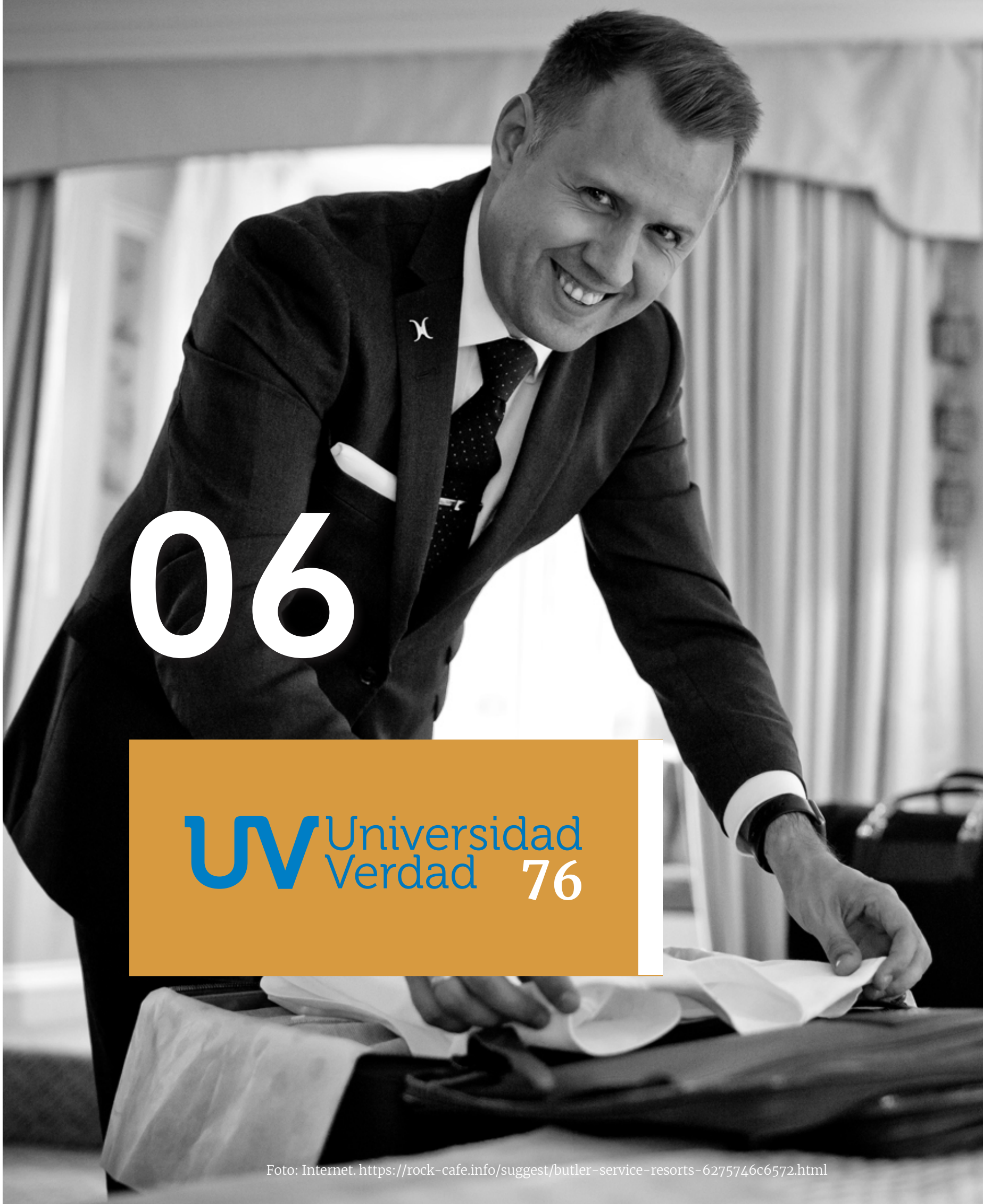




\section{ANÁLISIS DEL SISTEMA TURÍSTICO DE TRES CIUDADES PATRIMONIALES DE AMÉRICA: CUENCA, CUSCO Y PUEBLA}

\section{Analysis of the tourist system of three heritage cities in America: Cuenca, Cusco and Puebla}

Lcda. Ana Lucia Serrano PhD(c). Docente e investigadora del grupo de investigación empresarial y turístico de la Facultad de Ciencias de la Hospitalidad. Universidad de Cuenca ( Ecuador) (ana.serrano@ucuenca.edu.ec)

(https://orcid.org/0000-0002-1616-4144)

(iD Ing. Maria Elvira Farfán. Investigadora del grupo de investigación empresarial y turístico de la Facultad de Ciencias de la Hospitalidad. Universidad de Cuenca. (Ecuador) (elvifarfan@gmail.com) (https://orcid.org/0000-0002-2502-5664)

Joselyne Estefania Rodas Pesantez. Ayudante de Investigación del proyecto de airbnb de la Facultad de Ciencias de la Hospitalidad. Universidad de Cuenca. (Ecuador) (joselyne.rodasp97@ucuenca.edu.ec) (https://orcid.org/0000-0002-8910-6471)

Lcda. Elena Villafuerte Pucha. Docente e investigadora del Instituto Tecnológico Superior San Isidro. (Cuenca - Ecuador) (elenavillafuerte@sanisidro.edu.ec)

(https://orcid.org/0000-0002-3965-4218)

\section{Resumen}

La Organización de las Naciones Unidas para la Educación, la Ciencia y la Cultura, UNESCO, es una organización que, a partir del año 1978, ha creado la lista de patrimonios de la humanidad. Dentro de esta, se encuentran varios centros históricos que destacan por sus únicas edificaciones, con estilos arquitectónicos y artísticos únicos. Con base en esta lista, se ha decidido realizar esta investigación que busca analizar el sistema turístico de tres de estas ciudades declaradas como Patrimonio Cultural de la Humanidad y que pertenecen al continente americano, estas son: la ciudad de Cuenca-Ecuador, Cusco-Perú y Puebla-México. Para este análisis se ha optado por realizar un benchmarking de las ciudades en estudio, en primera instancia, como parte de los datos iniciales de la investigación se ha tomado en consideración el número de turistas que llegaron a las ciudades en el año 2016 siendo este el punto de partida para un estudio comparativo de los componentes de su sistema turístico (oferta, producto turístico, infraestructura, planta turística), con el fin de conocer por qué al ser ciudades con pares similares, no se han desarrollado de la misma manera dentro del ámbito turístico.

\section{Abstract}

The Organization of the United Nations for Education, Science and Culture, UNESCO, is an organization that since 1978 has created the list of the heritage of humanity, within it, there are several historical centers that stand out for its buildings, with unique architectural and artistic styles; this research is 
based on this list and it seeks to analyze the tourist system of three cities declared as cultural heritage of humanity that belong to the American continent, those cities are: Cuenca-Ecuador, Cusco-Peru and Puebla-Mexico. For this analysis, a benchmarking of the cities under study has been chosen. In the first instance, as part of the research gaps, the number of tourists that arrived in the cities in 2016 has been taken into consideration to use them as a starting point for a comparative study of the components of their tourism system (supply, tourist product, infrastructure, tourist facility) and realize why being cities with similar peers have not developed in the same way within the tourist field.

\section{Palabras clave}

Benchmarking, Ciudades patrimoniales, Sistema turístico, Oferta turística.

\section{Keywords}

Benchmarking, Heritage cities, Touristic system, Touristic Offer.

1.

\section{Introducción}

Actualmente, se considera al turismo como una actividad económica de gran importancia a nivel mundial. Esto, por su propiedad multiplicadora de empleos, por ser uno de los rubros más importantes dentro del producto interno bruto de los países en desarrollo y por su constante crecimiento percibido en el aumento de destinos turísticos (Organización Mundial de Turismo [OMT], 2005).

Dada su importancia en el desarrollo socio económico, nace el interés de analizar parte del sistema turístico de tres ciudades declaradas Patrimonio Cultural de la Humanidad por la Unesco, Cuenca-Ecuador, Cusco-Perú y Puebla-México. Se conoce en primera instancia que el sistema turístico está conformado por: demanda turística, oferta turística, superestructura, el producto y la planta turística (Boullón,
2006); sin la presencia de uno de estos elementos dentro de un destino, sería difícil lograr el desarrollo turístico dentro del mismo.

Para esta investigación de alcance exploratorio se ha optado por realizar un benchmarking de las ciudades y, por medio de una comparación inferencial, ha permitido analizar aspectos importantes dentro de las generalidades de las ciudades como: su ubicación, hidrografía, moneda, acceso, población y declaratorias. Dentro del producto turístico se analiza la arquitectura del lugar, sus declaratorias, la tipología de turismo que ofertan y sus asentamientos y, por último, dentro de la planta turística, el alojamiento y transporte aéreo, para analizar cómo estos elementos, que muestran pares similares entre la ciudades elegidas dentro de la investigación, inciden en el número de turistas, tanto nacionales como extranjeros, que llegan a las ciudades. Esto con el fin de identificar por qué, al ser ciudades que llevan una declaratoria patrimonial paralela, no cuentan con la misma afluencia de turistas.

\section{2.}

\section{Problemática y justificación}

Varios de los atractivos turísticos existentes en el mundo aún permanecen escondidos, esto sucede por una falta de planificación o simple falta de interés por explorar sus potencialidades a favor de la actividad turística (Monfort, 2000). 
Como parte de salvaguardar el patrimonio, la UNESCO ha nombrado, desde el año 1978, como Patrimonio de la Humanidad a aquellos sitios que contienen importancia tanto cultural como natural; la selección de aquellos lugares que forman parte de la lista de patrimonio se realiza con base en diez criterios. Se conoce que, hasta el año 2015, se han registrado 1031 sitios dentro de la lista, de los cuales: 802 son de orden cultural e histórico; 197, de carácter natural, y 32 que comparten ambas características. Esta denominación ha sido fundamental para la planificación turística para identificar, proteger, conservar y trasmitir el patrimonio; sin embargo, se puede ver, mediante un análisis de la demanda turística de ciertas ciudades patrimonio, que esta declaratoria no ha sido un factor relevante al momento de aumentar el número de visitantes y turistas que llegan al destino, sino, que en este caso, influye en gran medida en cómo se ha conformado todo el sistema turístico. En el caso de las tres ciudades elegidas para esta investigación, se conoce que en el año 2016, Cuenca-Ecuador ha recibido 290.160 turistas (Serrano et ál., 2018); Cusco-Perú, 305.279 turistas (Cantorán, 2018; Perú.com, 2017; PromPerú, 2017), y Puebla-México, 292.115,2 turistas (DataTur, 2017), tanto nacionales como extranjeros. Al tratarse de ciudades patrimoniales con características similares, deberían tener un número de entradas afín en un mismo año; sin embargo, en el caso de Cuenca no es así. Al presentar una demanda turística menor con relación a las otras dos ciudades, se considera urgente encontrar cuál es el problema dentro de la ciudad, que no permite este crecimiento en cifras turísticas tomando como base estas características similares entre ciudades.

\section{3.}

\section{Revisión teórica}

La actividad turística es altamente compleja por todos los elementos que intervienen en el momento de generar una experiencia a un visitante, por tanto, la planificación turística debe realizarse mediante colaboración entre la empresa pública y privada, con el fin de generar turismo sostenible en los destinos, es decir, un turismo responsable social, económica y ambientalmente. El papel del estado es fundamental, ya que es el organismo que se encarga de generar po- líticas públicas para el ordenamiento de la actividad, la generación de leyes, reglamentos y ordenanzas que permitan al sector privado tener las bases necesarias para poder emprender dentro del área turística. Para lograr la gestión turística adecuada, se debe partir del hecho de que un destino se desarrolla en un espacio geográfico determinado, el mismo que cuenta con componentes naturales como son: el clima, los ríos, las montañas, lagunas, entre otros; es decir, en términos turísticos, estaríamos hablando de los atractivos naturales de un lugar. Dentro de los componentes sociales, están las actividades humanas que se desarrollan en el lugar y que son generadoras de componentes culturales como son: las tradiciones, la religión, la lengua, gastronomía y otras; actividades que llegarían a ser los atractivos culturales, dentro de la planificación turística. Por otro lado, están los componentes económicos y políticos que son los que permiten el desarrollo de un lugar determinado (Cañizares, 2013). La materia prima del turismo son sus atractivos, ya sean naturales como culturales, y su presencia dentro de un territorio le da la denominación de espacio turístico (Boullón, 2006); y es en este espacio en donde se desarrolla el sistema turístico.

Para entender mejor el sistema turístico, se desarrollará una breve síntesis de cada uno de los elementos a analizar en el benchmarking de las ciudades. Se iniciará por la demanda que hace referencia a los consumidores o posibles consumidores de bienes y servicios (Molina et ál., 2007). Dentro de la planificación turística es de vital importancia realizar una caracterización de la demanda, para que la oferta pueda satisfacer las necesidades de los visitantes y cumplir con sus expectativas. El análisis estadístico de la demanda es de igual importancia, ya que el conocer la cantidad de turistas que recibe un lugar es el primer paso para identificar logros o fallas dentro de la planificación.

Por otra parte, la oferta turística, consiste en el conjunto de bienes y servicios que satisfacen a la demanda. Dentro del concepto de sistema turístico de Boullón (2006), se señala que el producto turístico está conformado por los mismos bienes y servicios que la oferta turística, por lo que se analizarán en conjunto, como los impulsadores del turismo, Dentro del benchmarking realizado con las ciudades de Cuenca, Cusco y Puebla, se han analizado las diferentes tipo- 
logías de turismo que se ofertan, como muestra de la diversidad de oferta dentro de las mismas.

Por otro lado, se ha hecho una investigación de los asentamientos humanos que han existido en las ciudades, los que han transformado a lo largo de los años la estructura de las ciudades, han impuesto estilos arquitectónicos únicos y han sido utilizados para la generación de productos como rutas temáticas. Un ejemplo de esto es la Ruta Francesa en la ciudad de Cuenca, que recorre aquellas casas con influencia europea en sus espacios y detalles, que obtuvieron gracias a los viajes que realizaba la población local para la exportación de la cascarilla [la cascarilla conocida como quina o cinchona es una planta que fue conocida como "el árbol de la vida", fue un producto de exportación entre 1775 y 1783 (Moya, 1994)] y los sombreros de paja toquilla (Fundación Municipal Turismo Para Cuenca, 2018).

La planta turística es quizá, uno de los componentes del sistema turístico más complejo y extenso, esta comprende los atractivos turísticos, el equipamiento e instalaciones, y la infraestructura.

En cuanto a los atractivos turísticos, dentro de este estudio, se ha realizado un cuadro de aquellos atractivos culturales y naturales relevantes de cada una de las ciudades, que aparecen como recomendación en más de una página web para viajeros y plataformas digitales (Lickorish, \& Jenkins, 1997).

La infraestructura es todo aquello que ha sido creado para satisfacer las necesidades de una población activa; es decir, dentro de esta, se tienen facilidades como vías de acceso, señalización, educación, salud, transporte, medios de comunicación, etc. Esta infraestructura que no ha sido creada pensando en los turistas, pero son grandes facilitadores para la actividad (Rozas y Sánchez, 2004). Dentro del turismo, juega un papel importante el acceso hacia los diferentes destinos, mediante la implementación de redes de transporte eficientes, tanto nacionales como internacionales; por tanto, se ha realizado un trabajo investigativo con respecto al transporte aéreo. Las tres ciudades en estudio cuentan con un aeropuerto, pero únicamente el de Cusco es internacional, por lo que para llegar a Cuenca y Puebla se deben realizar conexiones que varias veces encarecen los viajes y dificultan el acceso; por lo mismo se ha realizado un cuadro de resultados donde se puede ver qué aerolíneas vuelan hacia los destinos seleccionados, desde dónde lo hacen y qué escalas se requieren para llegar.

Los equipamientos se encuentran clasificados en alojamiento, alimentación, esparcimiento y otros servicios, como los de guías e intermediarios (Boullón, 2006). El alojamiento, por ejemplo, es indispensable dentro de la decisión de viaje y permanencia en un destino, por lo que se analiza la oferta de alojamiento a través de dos plataformas web: Booking y Expedia, para conocer el número de establecimientos y el rango de precios de estos de acuerdo con su categorización.

\section{4.}

\section{Metodología}

El estudio es de carácter cualitativo, con un alcance exploratorio. Por medio de la aplicación de un benchmarking, se realizó una comparación inferencial de los componentes del sistema turístico que poseen las ciudades patrimoniales (Cuenca, Cusco y Puebla), se identificaron las similitud entre éstas, basadas en los criterios por los que fueron declaradas Patrimonio de la Humanidad, y categorizadas como Patrimonio Cultural. Con base en la lista de la UNESCO, se conoce que la ciudad de Cuenca fue declarada como Patrimonio de la Humanidad bajo los criterios ii, iv y v; el Cusco, bajo los criterios iii y iv; y Puebla, bajo los criterios ii y iv. Es decir, las tres ciudades en estudio tienen como fundamento de patrimonialización al criterio número iv, que hace referencia a "ser uno de los ejemplos eminentes de procesos ecológicos y biológicos en el curso de la evolución de los ecosistemas" (Ajpis, 2015); y dos de ellas bajo el número ii de "testimoniar un importante intercambio de valores humanos a lo largo de un periodo de tiempo o dentro de un área cultural del mundo, en el desarrollo de la arquitectura, tecnología, artes monumentales, urbanismo o diseño paisajístico" (Ajpis, 2015).

Se ha realizado la revisión de literatura en diferentes portales web, como las páginas oficiales de turismo de cada una de las ciudades para recabar información clave sobre el sistema turístico de cada una; 
revisión de las estadísticas turísticas y boletines de oferta y demanda; además del uso de otras plataformas como: Booking y Expedia, para conocer cuál es la oferta de alojamiento que tienen dentro de las ciudades, con su categorización y con un rango de precios. La razón por la que se eligieron estas dos plataformas digitales para la obtención de información sobre alojamiento es porque Booking y Expedia son los principales canales de distribución en cuanto a reservas hoteleras, incluso por delante de las reservas directas a través de las propias páginas web de los establecimientos de alojamiento.

Para la obtención de información sobre vuelos, se ha optado por revisar frecuencias en las diferentes aerolíneas que permiten llegar hacia las tres ciudades en estudio, se tuvo como punto de partida a las ciudades de Nueva York, Buenos Aires y Ottawa, por ser las capitales de los países de donde, según el Boletín de indicadores turísticos del cantón Cuenca elaborado por el grupo de investigación de la Facultad Ciencias de la Hospitalidad de la Universidad de Cuenca en el año 2018, la ciudad de Cuenca recibe mayor número de turistas.

Para el análisis de resultados, se han construido tablas comparativas entre las ciudades.

\section{5.}

\section{Resultados}

A continuación, se presenta la primera tabla de resultados del benchmarking realizado entre las ciudades de Cuenca, Cusco y Puebla, Tabla 1.

Como se puede observar, esta tabla muestra, mediante una comparación, generalidades de las tres ciudades parte de este estudio. En primer lugar, se analiza su ubicación, en donde se observa que tanto Cuenca como Puebla, se encuentran en el sur y región sierra, con relación al país en el que se encuentran, Ecuador y México respectivamente; razón por la que comparten un clima similar y con paisajes similares.

La hidrografía de las tres ciudades en mención cuenta con ríos dentro de las mismas, y es, en todos los casos, parte de los atractivos turísticos importantes dentro de la ciudad. En relación con la moneda que se utiliza en cada una de las ciudades, se puede ver que Cusco, con el sol peruano, y Puebla, con el peso mexicano, sufren de inestabilidad macroeconómica debido a que su moneda se devalúa constantemente (UNWTo, 2017). Sin embargo, en términos turísticos, este aspecto ha provocado que los turistas, especialmente de Ecuador y de países que tienen como divisa el dólar, viajen y gasten más en el destino, ya que la devaluación las convierte en una moneda débil frente al dólar al momento de realizar cambio de divisas (Morantes, 2015).

En el caso de la ciudad de Cuenca, la realidad es otra, porque diferentes estudios indican que el precio del dólar afecta a la sociedad. La dolarización se lleva a cabo con el fin de evitar fluctuaciones y la devaluación de la moneda; el incremento del precio del dólar hace que para Ecuador sea beneficioso consumir en otros países de América, como Colombia, Perú y Venezuela, en donde no ha existido dolarización hasta el momento; sin embargo, esto hace que los turistas vean a la ciudad de Cuenca como un destino caro en relación al Cusco y Puebla (Vélez, 2015).

Las declaratorias resaltan el aspecto más importante y por el que fueron elegidas estas tres ciudades para el estudio, en este caso son declaradas Patrimonio Cultural de Humanidad por la UNESCO; cabe recalcar que Cusco, por ejemplo, cuenta con otras declaratorias como Capital Histórica del Perú (Municipalidad del Cusco, 2015) y tiene a Macchu Picchu declarado como la nueva maravilla del mundo moderno, méritos que son importantes al momento de promocionar en otros países a la ciudad, que en el año 2013 se posiciona en el segundo lugar en la campaña Ciudades Maravillas del Mundo (RPP Noticias, 2013); algo que, dicho por Moisés Bendezú Sandoval, presidente de la Asociación Campaña Cusco Ciudad Maravilla, ha ayudado a tener un incremento constante y permanente de turismo receptivo (OMT, 2005). 
Tabla 1

Generalidades

\begin{tabular}{|l|l|l|l|}
\hline & \multicolumn{1}{|c|}{ Cuenca, Ecuador } & \multicolumn{1}{c|}{ Cusco, Perú } & \multicolumn{1}{c|}{ Puebla, México } \\
\hline \multirow{2}{*}{ Ubicación } & $\begin{array}{l}\text { Sur del Ecuador - región } \\
\text { Sierra }\end{array}$ & Región Sur oriental de Perú & $\begin{array}{l}\text { Área este central de México, al } \\
\text { sureste de ciudad de México. }\end{array}$ \\
\hline Hidrografía & $\begin{array}{l}\text { Ríos: Tomebamba, Tarqui, } \\
\text { Machángara y Yanuncay. }\end{array}$ & $\begin{array}{l}\text { Ríos: Ucubamba, Apurí- } \\
\text { mac, Ene, Inambari e Inuya. }\end{array}$ & $\begin{array}{l}\text { Ríos: Atoyac, Nexapa, Tuxpan, } \\
\text { Necaxa, Cazones y Tehuacán. }\end{array}$ \\
\hline Moneda & Dólar estadounidense & Sol peruano & Peso mexicano \\
\hline \multirow{2}{*}{ Declaratorias } & $\begin{array}{l}\text { Patrimonio cultural de la la } \\
\text { humanidad (1999). }\end{array}$ & Capital histórica del Perú. & $\begin{array}{l}\text { Patrimonio Cultural de la Hu- } \\
\text { manidad, Unesco (1987). }\end{array}$ \\
\cline { 2 - 5 } & & $\begin{array}{l}\text { Patrimonio Cultural de la } \\
\text { Humanidad (1983). }\end{array}$ & $\begin{array}{l}\text { Mejor destino de Latinoamé- } \\
\text { rica, 2017 }\end{array}$ \\
\cline { 2 - 5 } & & $\begin{array}{l}\text { Nueva maravilla del mundo } \\
\text { moderno. }\end{array}$ & \\
\hline
\end{tabular}

Fuente: Adaptado a partir de la revisión bibliográfica realizada en los sitios web oficiales de las tres ciudades:

Cuenca (Municipio de Cuenca, 2016), Cusco (ENPERÚ, 2015), y Puebla (Rengifo, 2017).

\subsection{Oferta y producto turístico}

La Tabla 2 muestra parte de la oferta y productos turísticos de las tres ciudades. En primera instancia, se analiza la arquitectura que tiene influencia de aquellos asentamientos humanos que han existido en las ciudades a través de los años. En este sentido Cuenca, Cusco y Puebla fueron colonizadas por los españoles, razón por la que cuentan con estilos arquitectónicos similares, como son: las construcciones coloniales, la influencia de la religión católica y, por ende, la presencia de grandes iglesias y catedrales, una plaza de armas o parque central y la estructura de las calles en Damero. Características por la que sus centros históricos han sido declarados como Patrimonio de la Humanidad por la UNESCO. 


\section{Tabla 2}

Análisis de la oferta y producto turístico

\begin{tabular}{|c|c|c|c|}
\hline & Cuenca, Ecuador & Cusco, Perú & Puebla, México \\
\hline Arquitectura & $\begin{array}{l}\text { Estilo de Damero en las ca- } \\
\text { lles, construcciones colo- } \\
\text { niales, republicanas. }\end{array}$ & $\begin{array}{l}\text { Palacios y templos del Imperio } \\
\text { Inca, construcciones colonia- } \\
\text { les muros de transición (arqui- } \\
\text { tectura incaica y colonial). }\end{array}$ & $\begin{array}{l}\text { Arquitectura Colonial, cons- } \\
\text { trucciones barrocas, arte pre- } \\
\text { hispánico y renacentista. }\end{array}$ \\
\hline Asentamientos & $\begin{array}{l}\text { Asentamientos Cañaris e In- } \\
\text { cas. Conquista española. }\end{array}$ & $\begin{array}{l}\text { Centro político y territorial } \\
\text { Inca. Conquista española }\end{array}$ & $\begin{array}{l}\text { Asentamientos mesoamerica- } \\
\text { nos. Culturas pre-hispánicas: } \\
\text { Totonaca, Mixtexas, Otomíes, } \\
\text { Nahuas, Mexicas o Aztecas, } \\
\text { Cholultecas, Huejotzincas y } \\
\text { Popolocas. Conquista española }\end{array}$ \\
\hline $\begin{array}{l}\text { Tipologías de } \\
\text { turismo }\end{array}$ & $\begin{array}{l}\text { Turismo cultural } \\
\text { Turismo religioso } \\
\text { Ecoturismo } \\
\text { Turismo rural } \\
\text { Turismo vivencial }\end{array}$ & $\begin{array}{l}\text { Turismo cultural } \\
\text { Turismo de deporte } \\
\text { Ecoturismo } \\
\text { Turismo gastronómico } \\
\text { Turismo religioso }\end{array}$ & $\begin{array}{l}\text { Ecoturismo } \\
\text { Turismo religioso } \\
\text { Turismo cultural } \\
\text { Turismo gastronómico } \\
\text { Turismo arquitectónico } \\
\text { Turismo arqueológico }\end{array}$ \\
\hline Rutas/ tours & $\begin{array}{l}\text { Ruta de San Joaquín y el río } \\
\text { Yanuncay (turismo rural) } \\
\text { Ruta de los molinos y el pan } \\
\text { Ruta vivencial, artesanías } \\
\text { cuencanas. } \\
\text { Ruta de los museos } \\
\text { Ruta Francesa } \\
\text { Ruta Artesanal } \\
\text { Ruta de Aventura } \\
\text { Ruta de la espiritualidad } \\
\text { Ruta de los museos y edifi- } \\
\text { caciones patrimoniales } \\
\text { Ruta de los sabores } \\
\text { Ruta de naturaleza y bien- } \\
\text { estar } \\
\text { Ruta vida cotidiana } \\
\text { Recorrido bus turístico }\end{array}$ & $\begin{array}{l}\text { Recorrido por la plaza mayor } \\
\text { Recorrido por el pintoresco } \\
\text { barrio de San Blas } \\
\text { Visita a fortalezas Incas } \\
\text { Tour a Macchu Picchu } \\
\text { Tour hacia Sacsayhuaman, } \\
\text { Qoricancha, Qenko y Tambo- } \\
\text { machay. } \\
\text { City tour } \\
\text { Tour al último puente Inca, } \\
\text { Q'eswachaka } \\
\text { Descubre la laguna Humantay } \\
\text { Caminata montaña de } 7 \text { colo- } \\
\text { res } \\
\text { Cusco, Valle Sagrado y Macchu } \\
\text { Picchu } \\
\text { Comida casera peruana }\end{array}$ & $\begin{array}{l}\text { Visita Puebla y Cholula en } 1 \text { día } \\
\text { Tour a Zacatlán de las man- } \\
\text { zanas } \\
\text { Tour de Chautla hacienda } \\
\text { Tour de patrimonio } \\
\text { Tour culinario } \\
\text { Tour por casco central en tran- } \\
\text { vía de dos pisos } \\
\text { Tour en bus panorámico por el } \\
\text { pueblo mágico de Cholula } \\
\text { Tour a Atlixco de las flores } \\
\text { Tour a pie por la arquitectura } \\
\text { de Puebla } \\
\text { Tour gastronómico con catas } \\
\text { Senderismo hacia el Parque } \\
\text { Nacional Izta-Popo }\end{array}$ \\
\hline Sitio web & $\begin{array}{l}\text { http://cuencaecuador.com. } \\
\text { ec/ }\end{array}$ & $\begin{array}{l}\text { https://www.cuscoperu.com/ } \\
\text { es/viajes/cusco }\end{array}$ & http://puebla.travel/es/puebla \\
\hline
\end{tabular}

Fuente: Adaptado a partir de la revisión bibliográfica realizada en los sitios web oficiales de las tres ciudades

(El Comercio, 2016; Get your Guide, 2018; Vizcarra, 2014). 


\subsection{Planta turística y atractivos turísticos}

En las tipologías de turismo que se ofertan en las ciudades se puede ver la similitud con respecto a la factibilidad para realizar turismo cultural y religioso por la belleza de sus construcciones y organización de sus centros históricos, diversificación en otros tipos de propuestas, que caracterizan a cada uno de los destinos y que ha permitido el desarrollo y creación de diversas rutas, tours y paquetes turísticos dentro de las ciudades y hacia atractivos cercanos.
En las ciudades de Cuenca y Puebla, se puede ver, esta diversificación dentro de la oferta, al existir propuestas de turismo rural, vivencial, espiritual, de patrimonio, gastronómico y más; sin embargo, la realidad de Cusco, resulta ser diferente debido a que gran parte de su oferta gira en torno a Macchu Picchu, que es el lugar de destino turístico de mayor importancia; si bien este sitio genera grandes ingresos para el país, también limita el desarrollo de nuevas propuestas dentro del casco histórico de la ciudad. El detalle se puede visualizar en la Tabla 3.

\section{Tabla 3}

Atractivos turísticos culturales y naturales

\begin{tabular}{|c|c|c|c|}
\hline \multicolumn{4}{|c|}{ Planta turística } \\
\hline $\begin{array}{l}\text { Atractivos } \\
\text { culturales }\end{array}$ & $\begin{array}{l}\text { Ruinas arqueológicas de } \\
\text { Todo Santos } \\
\text { Pumapungo } \\
\text { Catedral de la Inmaculada } \\
\text { Catedral Vieja } \\
\text { San Sebastián } \\
\text { San Blas } \\
\text { El Barranco } \\
\text { Plaza de las Flores } \\
\text { Turi }\end{array}$ & $\begin{array}{l}\text { Choquequirao } \\
\text { Sacsayhuaman } \\
\text { Qenko } \\
\text { Tambomachay } \\
\text { Ollantaytambo } \\
\text { Macchu Picchu } \\
\text { Barrio de San Blas } \\
\text { Paucartambo } \\
\text { Catedral de Cusco } \\
\text { Plaza de Armas } \\
\text { Templo de la Compañía de Jesús. }\end{array}$ & $\begin{array}{l}\text { Catedral } \\
\text { Centro histórico } \\
\text { El Parián } \\
\text { La calle de los dulces } \\
\text { Museo Internacional Barroco } \\
\text { Mercado Melchor Ocampo } \\
\text { Plazoleta de los Sapos } \\
\text { Barrio del Artista } \\
\text { Capilla del Rosario } \\
\text { Zona arqueológica de Cantona }\end{array}$ \\
\hline $\begin{array}{l}\text { Atractivos } \\
\text { naturales }\end{array}$ & $\begin{array}{l}\text { Orquideario, Universidad } \\
\text { de Cuenca } \\
\text { Parque Nacional Cajas } \\
\text { Río Tomebamba }\end{array}$ & $\begin{array}{l}\text { Valle Sagrado } \\
\text { Maras (minas de sal) }\end{array}$ & $\begin{array}{l}\text { Parque Nacional Iztac- } \\
\text { cíhuatl-Popocatépetl } \\
\text { Biznaga gigante } \\
\text { Cholula } \\
\text { Acolhua } \\
\text { Atlitán } \\
\text { Agroparque la Esperanza } \\
\text { Barranca el Tabaco }\end{array}$ \\
\hline
\end{tabular}

Fuente: Adaptado a partir de la revisión bibliográfica realizada en los sitios web oficiales de las tres ciudades (Dejtiar, 2017). 


\subsection{Oferta de alojamiento en las plataformas digita- les: Booking y Expedia}

Existen varios sitios en internet en donde se puede leer información acerca de los atractivos culturales y naturales de un lugar determinado. Para la realización de la Tabla 3, se realizó una revisión de listas de atractivos en la página oficial de turismo de cada una de las ciudades y en Trip Advisor, con el fin de conocer cuáles son los lugares que más se promocionan dentro de las mismas y que forman parte de su planta turística y de su patrimonio como ciudad.

Al tratarse de ciudades Patrimonio Cultural de la Humanidad, su lista de atractivos culturales es más amplia con relación a los naturales; y, en su mayoría, se trata de iglesias y edificaciones de la época de la colonia en adelante, que muestran la fachada actual de sus centros históricos, los tres con influencia en estilos estéticos europeos. Se ha realizado un cuadro comparativo en el que se puede ver claramente el número de establecimientos de alojamiento que se oferta en cada una de las ciudades de estudio a través de las plataformas digitales Booking y Expedia; y a continuación, se presenta esta información en la Tabla 4.

\section{Tabla 4}

Oferta de alojamiento en las plataformas digitales: Booking y Expedia

\begin{tabular}{|l|l|l|l|l|l|l|}
\hline Ciudades & $\begin{array}{c}\text { Número de es- } \\
\text { tablecimientos } \\
\text { Booking }\end{array}$ & $\begin{array}{c}\text { Número de es- } \\
\text { tablecimientos } \\
\text { Expedia }\end{array}$ & $\begin{array}{c}\text { Precio más } \\
\text { bajo Booking/ } \\
\text { Dólares }\end{array}$ & $\begin{array}{c}\text { Precio más } \\
\text { alto Booking } \\
\text { / Dólares }\end{array}$ & $\begin{array}{l}\text { Precio más } \\
\text { bajo Expe- } \\
\text { dia/ Dólares }\end{array}$ & $\begin{array}{c}\text { Precio más } \\
\text { alto Expe- } \\
\text { dia/ Dólares }\end{array}$ \\
\hline $\begin{array}{l}\text { Cuenca, } \\
\text { Ecuador }\end{array}$ & 125 & 115 & 5 & 103 & 8 & 316 \\
\hline $\begin{array}{l}\text { Pueb la }, \\
\text { México }\end{array}$ & 162 & 173 & 7 & 250 & 7 & 270 \\
\hline $\begin{array}{l}\text { Cu s c o } \\
\text { Perú }\end{array}$ & 953 & 494 & 5 & 895 & 5 & 995 \\
\hline
\end{tabular}

Fuente: Adaptado a partir de la información obtenida en el mes de marzo, 2017 en las plataformas digitales: Booking y Expedia (El Economista, 2018). 


\section{Figura 1}

Número de establecimientos ofertados en Booking y Expedia

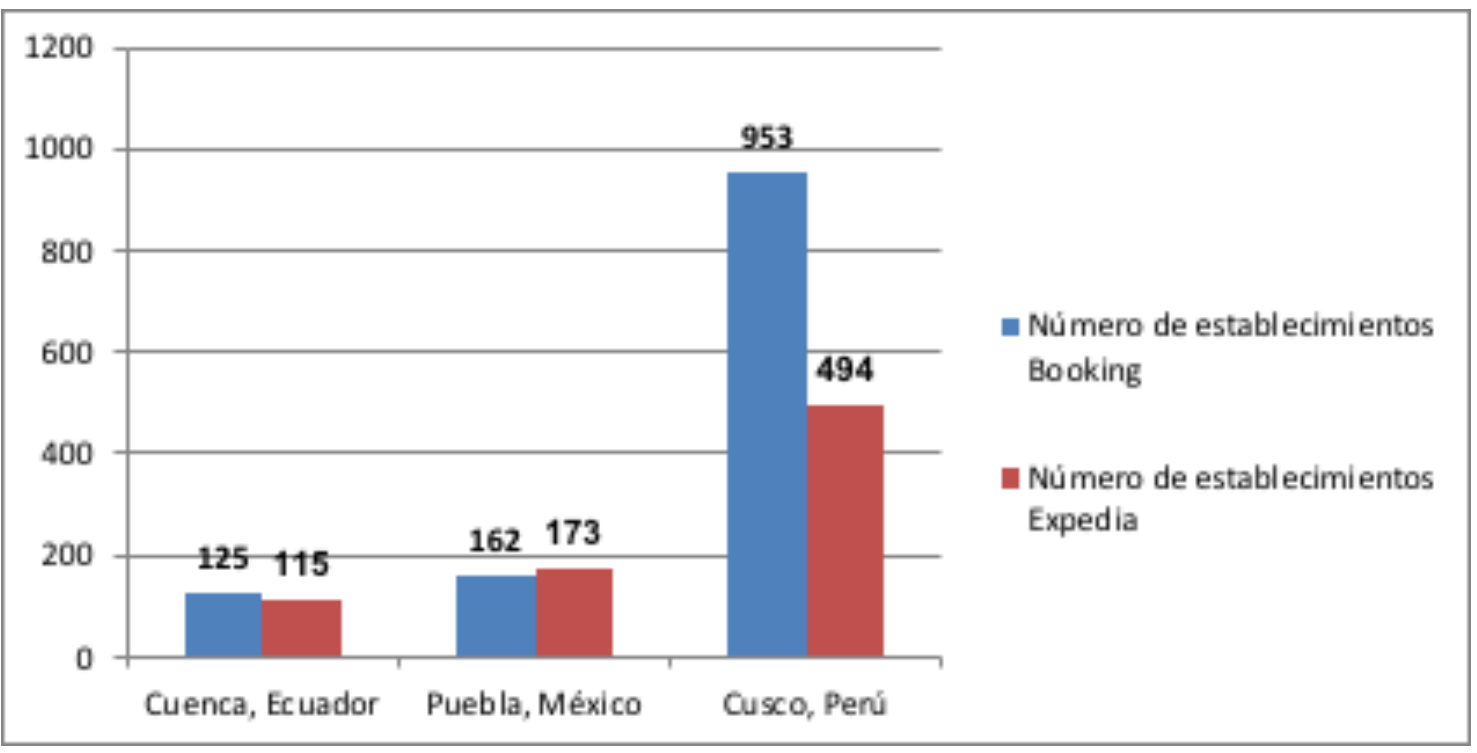

Fuente: Adaptado con base en la información obtenida en el mes de marzo, 2017; (Booking, s.f.; Expedia, s.f.)

En el caso de la Figura 1, la ciudad del Cusco cuenta con una oferta de alojamiento más amplio en las dos plataformas, con relación a Cuenca que es la ciudad que cuenta con menor oferta. Si se analiza con base en el número de turistas que llegan a estas dos ciudades, en el año 2016 sabemos que la diferencia es bastante alarmante; Cuenca-Ecuador ha recibido 290,160 turistas (Serrano et ál., 2018) a diferencia de Cusco-Perú que ha recibido 3053.279 turistas (PromPerú, 2017) es decir un 90,45\% más de turistas; por tanto, su oferta debería ser más grande en cantidad y más diversificada en cuanto a categorización y rango de precios.

Al analizar este dato de la demanda turística en Cusco con relación al número de turistas que llegan a Puebla (3 630,530 turistas) (DataTur, 2017), en el mismo año 2016 se puede ver que es aún mayor, pero que no ofertan ni un 50\% de establecimientos con relación al Cusco, tal vez una señal de que la segunda ciudad en mención no ha realizado un análisis adecuado y que existe sobreoferta de alojamiento.

En la Figura 2 se puede observar el precio más bajo y alto de los establecimientos de alojamiento ofertados en cada una de las ciudades. En Cusco, Perú se observa la oferta de alojamiento más diversificada con precios que van desde los $\$ 5$ hasta los $\$ 995$, lo que muestra que turistas con diferentes condiciones económicas pueden acceder a servicios de pernoctación en el lugar. 
Figura 2

Número de establecimientos ofertados en Booking y Expedia por análisis mediante tarifas

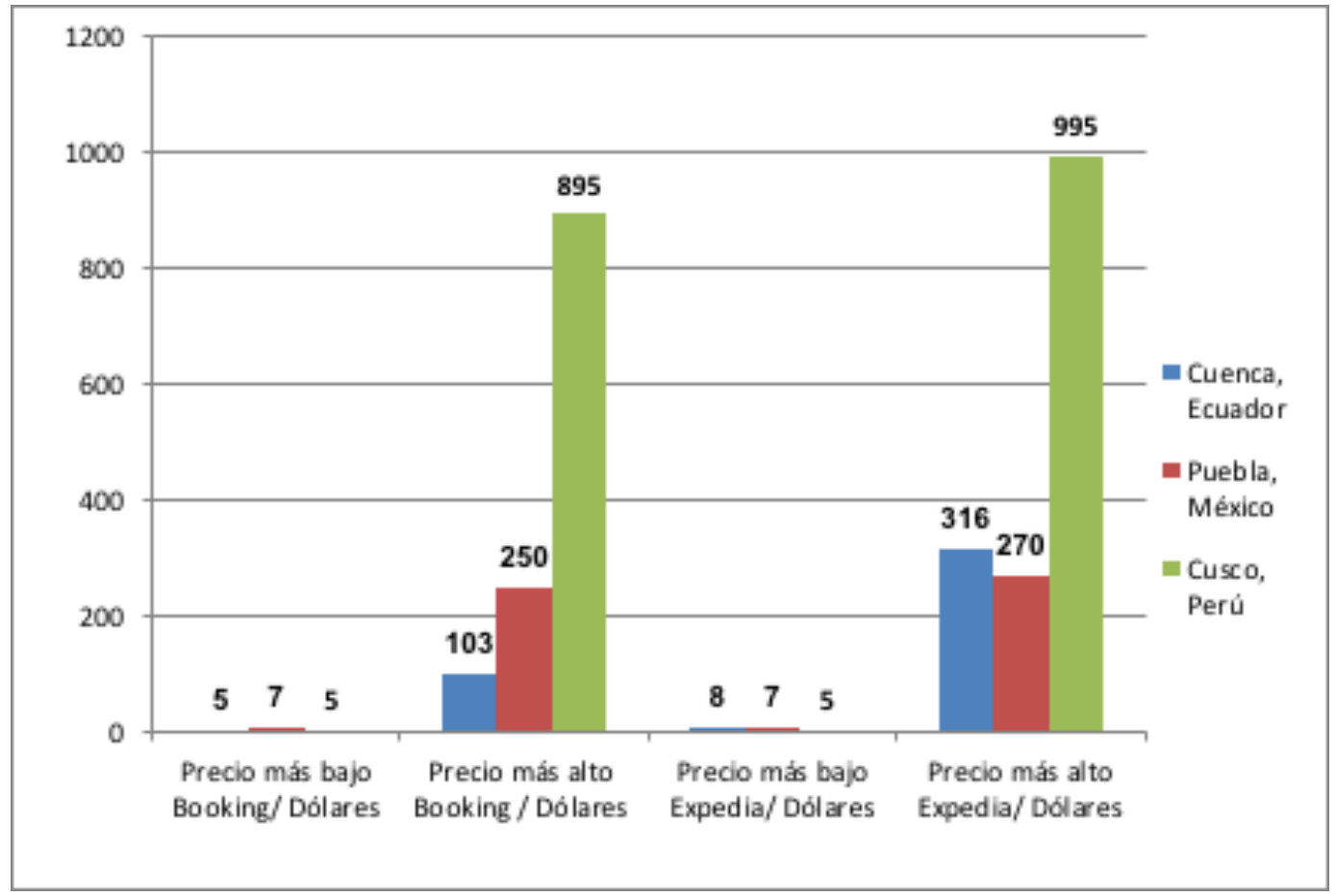

Fuente: Adaptado con base en la información obtenida en el mes de marzo, 2017; (Booking, s.f.; Expedia, s.f.)

\subsection{Categorización del alojamiento en Cuenca - Ecuador según Booking y Expedia}

En las Tablas 5, 6 y 7, se puede ver con mayor detalle cómo se encuentran categorizados los establecimientos de alojamiento por ciudad y portal web, cada una de estas categorías con un rango de precios desde el más bajo al más alto; lo que se debería saber es, si en cada una de las ciudades presentadas, existe un control para asegurar la relación precio-calidad, de acuerdo con la categoría de los establecimientos.

Dentro de la planta turística de un lugar se encuentra implícita la infraestructura, y en el caso de este estudio investigativo, se analizará uno de sus elementos que es el transporte aéreo. A continuación, se mostrarán cuadros para conocer mediante qué aerolíneas y con qué escalas se puede llegar a las tres ciudades, tomando como punto de partida las ciudades: Nueva York, Buenos Aires y Ottawa; al ser las capitales de los 3 países de mayor procedencia de turistas hacia la ciudad de Cuenca, esto de acuerdo al Boletín de indicadores turísticos del cantón Cuenca, realizado por el Departamento de Investigación de la Facultad Ciencias de la Hospitalidad de la Universidad de Cuenca y publicado en marzo 2018. 


\section{Tabla 5}

Categorización del alojamiento en Cuenca

\begin{tabular}{|c|c|c|c|c|}
\hline \multicolumn{5}{|c|}{$\begin{array}{c}\text { Planta turística } \\
\text { Alojamiento Cuenca }\end{array}$} \\
\hline \multicolumn{2}{|c|}{ Establecimientos localizados } & Categoría & Cantidad & Precios \\
\hline \multirow{6}{*}{ Booking } & \multirow{6}{*}{$\begin{array}{c}125 \\
\text { establecimientos }\end{array}$} & Hoteles 2 estrellas & 2 & $\$ 16-\$ 17$ \\
\hline & & Hoteles 3 estrellas & 7 & $\$ 15-\$ 40$ \\
\hline & & Hoteles 4 estrellas & 20 & $\$ 28-\$ 80$ \\
\hline & & Hoteles 5 estrellas & 2 & $\$ 63-\$ 103$ \\
\hline & & Hostales & 44 & $\$ 5-\$ 25$ \\
\hline & & Departamentos & 13 & $\$ 20-\$ 98$ \\
\hline \multirow{5}{*}{ Expedia } & \multirow{5}{*}{$\begin{array}{c}115 \\
\text { establecimientos }\end{array}$} & Hoteles 3 estrellas & 47 & $\$ 69-\$ 162$ \\
\hline & & Hoteles 4 estrellas & 7 & $\$ 64-\$ 316$ \\
\hline & & Departamento & 15 & $\$ 37-\$ 53$ \\
\hline & & Bed and breakfast & 9 & $\$ 24-\$ 56$ \\
\hline & & Aparthotel & 5 & $\$ 8-\$ 55$ \\
\hline
\end{tabular}

Fuente: Adaptado a partir de la información obtenida en el mes de marzo, 2017 en las plataformas digitales: Booking y Expedia. 


\section{Tabla 6}

Categorización de alojamiento en Cusco

\begin{tabular}{|c|c|c|c|c|}
\hline \multicolumn{5}{|c|}{$\begin{array}{c}\text { Planta turística } \\
\text { Alojamiento Cuenca }\end{array}$} \\
\hline \multicolumn{2}{|c|}{ Establecimientos localizados } & Categoría & Cantidad & Precios \\
\hline \multirow{10}{*}{ Booking } & \multirow{10}{*}{$\begin{array}{c}953 \\
\text { establecimientos }\end{array}$} & Albergues & 97 & $\$ 5-\$ 50$ \\
\hline & & Habitaciones en casas particulares & 84 & $\$ 5-\$ 120$ \\
\hline & & Bed and breakfast, hostales & 334 & $\$ 5-\$ 150$ \\
\hline & & Hoteles 1 estrella & 8 & $\$ 12-\$ 48$ \\
\hline & & Hoteles 2 estrellas & 45 & $\$ 8-\$ 72$ \\
\hline & & Hoteles 3 estrellas & 105 & $\$ 20-\$ 151$ \\
\hline & & Hoteles 4 estrellas & 17 & $\$ 66-\$ 179$ \\
\hline & & Hoteles 5 estrellas & 8 & $\$ 142-\$ 895$ \\
\hline & & Hostales & 44 & $\$ 5-\$ 25$ \\
\hline & & Departamentos & 13 & $\$ 20-\$ 98$ \\
\hline \multirow{6}{*}{ Expedia } & \multirow{6}{*}{$\begin{array}{c}494 \\
\text { establecimientos }\end{array}$} & Bed and breakfast & 26 & $\$ 16-\$ 60$ \\
\hline & & Apart-hotel & 10 & $\$ 18-\$ 99$ \\
\hline & & Hostel & 62 & $\$ 5-\$ 50$ \\
\hline & & Hoteles 3 estrellas & 149 & $\$ 23-\$ 350$ \\
\hline & & Hoteles 4 estrellas & 13 & $\$ 84-\$ 252$ \\
\hline & & Hoteles 5 estrellas & 4 & $\$ 147-\$ 995$ \\
\hline
\end{tabular}

Fuente: Adaptado a partir de la información obtenida en el mes de marzo, 2018 en las plataformas digitales: Booking y Expedia. 
Tabla 7

Categorización de alojamiento en Puebla

\begin{tabular}{|c|c|c|c|c|}
\hline \multicolumn{5}{|c|}{$\begin{array}{c}\text { Planta turística } \\
\text { Alojamiento Cuenca }\end{array}$} \\
\hline \multicolumn{2}{|c|}{ Establecimientos localizados } & Categoría & Cantidad & Precios \\
\hline \multirow{6}{*}{ Booking } & \multirow{6}{*}{$\begin{array}{c}162 \\
\text { establecimientos }\end{array}$} & Hoteles 2 estrellas & 7 & $\$ 9-\$ 56$ \\
\hline & & Hoteles 3 estrellas & 32 & $\$ 9-\$ 45$ \\
\hline & & Hoteles 4 estrellas & 57 & $\$ 19-\$ 203$ \\
\hline & & Hoteles 5 estrellas & 15 & $\$ 60-\$ 250$ \\
\hline & & Hostales & 13 & $\$ 7-\$ 61$ \\
\hline & & Departamentos & 10 & $\$ 20-\$ 65$ \\
\hline \multirow{6}{*}{ Expedia } & \multirow{6}{*}{$\begin{array}{c}173 \\
\text { establecimientos }\end{array}$} & Hoteles 3 estrellas & 91 & $\$ 20-\$ 63$ \\
\hline & & Hoteles 4 estrellas & 19 & $\$ 55-\$ 249$ \\
\hline & & Hoteles 5 estrellas & 2 & $\$ 113-\$ 270$ \\
\hline & & Hostales & 13 & $\$ 7-\$ 30$ \\
\hline & & Apart hotel & 4 & $\$ 11-\$ 56$ \\
\hline & & Departamento & 7 & $\$ 11-95$ \\
\hline
\end{tabular}

Fuente: Adaptado a partir de la información obtenida en el mes de marzo, 2018 en las plataformas digitales:

Booking y Expedia.

Lo que muestran las Tablas 8, 9 y 10 es que es fácil llegar hacia los destinos en estudio mediante transporte aéreo, al ser amplio el número de aerolíneas que lo permiten. Algo que significa un problema en Puebla y Cuenca es la no presencia de un aeropuerto internacional, además de otros inconvenientes como el suceso ocurrido en Cuenca el 19 de agosto de 2016, que por fallas en la pista de aterrizaje, se cerró el aeropuerto Mariscal Lamar y causó ciertos inconvenientes con el número de vuelos de conexión que se cancelaron, afectando así al turismo y, al mismo tiempo, a los propietarios de diferentes establecimientos de alojamiento, operadoras, alimentos y bebidas, entre otros. A partir de esto, Tame notificó que no logran generar demanda y, por tanto, los ingresos esperados con sus operaciones a Cuenca. En el mes de abril del 2017, deciden dejar de operar en la ruta Cuenca-Guayaquil-Cuenca. 


\section{Tabla 8}

Aerolíneas que vuelan desde Nueva York hasta Cuenca, Cusco y Puebla

\begin{tabular}{|c|c|c|}
\hline $\begin{array}{c}\text { Vuelos desde } \\
\text { Nueva York } \\
\text { Estados Unidos }\end{array}$ & Aerolíneas & Escalas \\
\hline \multirow{8}{*}{$\begin{array}{l}\text { Nueva York - } \\
\text { Quito - } \\
\text { Nueva York }\end{array}$} & Tame & Directo \\
\hline & Aeroméxico & Ciudad de México \\
\hline & United Airlines & Houston-Bogotá \\
\hline & American Airlines & Miami \\
\hline & LATAM & Ciudad de México \\
\hline & Copa Airlines & Panamá \\
\hline & Delta Airlines & Atlanta \\
\hline & & $\begin{array}{l}\text { * La ciudad de Cuenca no cuenta con aeropuerto inter- } \\
\text { nacional, todos los vuelos se conectan internamente a } \\
\text { través de Tame y LATAM. }\end{array}$ \\
\hline \multirow{4}{*}{$\begin{array}{l}\text { Nueva York - } \\
\text { Cusco - } \\
\text { Nueva York }\end{array}$} & $\begin{array}{l}\text { Avianca (hasta } \\
\text { marzo de 2020) }\end{array}$ & Bogotá-Lima-Medellín \\
\hline & LATAM & Lima-Miami- Dallas \\
\hline & American Airlines & Miami-Dallas-Lima \\
\hline & United Airlines & Lima-Bogotá \\
\hline \multirow{5}{*}{$\begin{array}{l}\text { Nueva York - } \\
\text { México DF - } \\
\text { Nueva York }\end{array}$} & Delta Air Lines & Detroit-Atlanta-Directo \\
\hline & United Airlines & Houston- Chicago \\
\hline & Aeroméxico & Directo \\
\hline & American Airlines & Dallas \\
\hline & & $\begin{array}{l}\text { * La ciudad de Puebla no cuenta con aeropuerto inter- } \\
\text { nacional, todos los vuelos se conectan internamente } \\
\text { desde Monterrey a través de Aeroméxico }\end{array}$ \\
\hline
\end{tabular}

Fuente: Adaptado a partir de la información obtenida en el mes de mayo, 2018 en Despegar.com 


\section{Tabla 9}

Aerolíneas que vuelan desde Buenos Aires hasta Cuenca, Cusco y Puebla

\begin{tabular}{|c|c|c|}
\hline $\begin{array}{l}\text { Vuelos desde } \\
\text { Buenos Aires, } \\
\text { Argentina }\end{array}$ & Aerolíneas & Escalas \\
\hline \multirow{4}{*}{$\begin{array}{l}\text { Buenos Aires - } \\
\text { Quito - } \\
\text { Buenos Aires }\end{array}$} & Avianca & Lima-Bogotá-Guayaquil \\
\hline & LATAM & Lima-Santiago de Chile-Córdoba \\
\hline & Copa Airlines & Panamá-Lima-Bogotá \\
\hline & & $\begin{array}{l}\text { * La ciudad de Cuenca no cuenta con aeropuerto inter- } \\
\text { nacional, todos los vuelos se conectan internamente a } \\
\text { través de Tame y LATAM. }\end{array}$ \\
\hline \multirow{2}{*}{$\begin{array}{l}\text { Buenos Aires - } \\
\text { Cusco - } \\
\text { Buenos Aires }\end{array}$} & Avianca & Lima- Bogotá- Arequipa-Juliaca \\
\hline & LATAM & $\begin{array}{l}\text { Asunción-Lima-Santiago de Chile-Córdoba- San } \\
\text { Miguel de Tucumán }\end{array}$ \\
\hline \multirow{9}{*}{$\begin{array}{l}\text { Buenos Aires - } \\
\text { México DF - } \\
\text { Buenos Aires }\end{array}$} & LATAM & Santiago de Chile- Lima- Sao Paulo \\
\hline & United Airlines & Houston- Nueva York \\
\hline & Aeroméxico & Directo \\
\hline & Delta Air Lines & Atlanta- Los Ángeles \\
\hline & Avianca & Lima- San Salvador- Bogotá- Panamá \\
\hline & Copa Airlines & Panamá \\
\hline & American Airlines & Miami- Dallas \\
\hline & $\begin{array}{l}\text { Aerolíneas } \\
\text { Argentinas }\end{array}$ & Cancún- Directo \\
\hline & & $\begin{array}{l}\text { *La ciudad de Puebla no cuenta con aeropuerto inter- } \\
\text { nacional, todos los vuelos se conectan internamente } \\
\text { desde Monterrey y México DF a través de Aeroméxico. }\end{array}$ \\
\hline
\end{tabular}

Fuente: Adaptado a partir de la información obtenida en el mes de mayo, 2018 en Despegar.com 


\section{Tabla 10}

Aerolíneas que vuelan desde Ottawa hasta Cuenca, Cusco y Puebla

\begin{tabular}{|c|c|c|}
\hline $\begin{array}{c}\text { Vuelos desde } \\
\text { Ottawa, Canadá }\end{array}$ & Aerolíneas & Escalas \\
\hline \multirow{7}{*}{$\begin{array}{l}\text { Ottawa - } \\
\text { Quito - } \\
\text { Ottawa }\end{array}$} & American Airlines & Filadelfia- Miami \\
\hline & United Airlines & Nueva York- Guayaquil- Houston- Washington \\
\hline & Air Canada & Nueva York- Houston- Toronto \\
\hline & Delta Air Lines & Atlanta- Nueva York- Detroit- Washington \\
\hline & Copa Airlines & Chicago- Panamá- Washington \\
\hline & Tame & Nueva York \\
\hline & & $\begin{array}{l}\text { *La ciudad de Cuenca no cuenta con aeropuerto inter- } \\
\text { nacional, todos los vuelos se conectan internamente a } \\
\text { través de Tame y LATAM. }\end{array}$ \\
\hline \multirow{5}{*}{$\begin{array}{l}\text { Ottawa - } \\
\text { Cusco - } \\
\text { Ottawa }\end{array}$} & American Airlines & Filadelfia- Dallas- Lima- Miami- Nueva York \\
\hline & United Airlines & Nueva York- Lima- Orlando- Washington \\
\hline & Air Canada & Boston- Miami- Lima- Nueva York- Toronto \\
\hline & Avianca & Nueva York- Lima- Toronto \\
\hline & LATAM & Boston- Orlando- Washington- Lima- Nueva York \\
\hline \multirow{6}{*}{$\begin{array}{l}\text { Ottawa - } \\
\text { México DF - } \\
\text { Ottawa }\end{array}$} & Delta Air Lines & Nueva York- Toronto- Detroit- Monterrey \\
\hline & United Airlines & Washington- Houston- Chicago \\
\hline & Air Canada & Toronto \\
\hline & American Airlines & Filadelfia- Dallas \\
\hline & Aeroméxico & Toronto \\
\hline & & $\begin{array}{l}\text { *La ciudad de Puebla no cuenta con aeropuerto inter- } \\
\text { nacional, todos los vuelos se conectan internamente } \\
\text { desde Monterrey y México DF a través de Aeroméxico. }\end{array}$ \\
\hline
\end{tabular}

Fuente: Adaptado a partir de la información obtenida en el mes de mayo, 2018 en Despegar.com

\section{6.}

\section{Conclusiones}

Las ciudades que han sido declaradas patrimonio deberían tener mayor oportunidad para ofertar el turismo de su zona, pero la realidad es otra, por el hecho de que no se aprovechan los recursos turísticos para mantener el gran potencial en atractivos. Solo ciertas ciudades han logrado sobresalir en el turismo y esto genera cierta entrada económica. En el caso de Puebla y Cusco, son dos ciudades que han implementado es- trategias para atraer al turismo y, sobre todo, aprovechan el hecho de ser ciudades patrimonio. Una de las ventajas que llevan ciertos países es que poseen una planta turística funcional $y$, sobre todo, el movimiento de marketing es más amplio, a diferencia del caso de la ciudad de Cuenca - Ecuador, que en los últimos años ha tenido ciertas bajas en el turismo, pese a que es una ciudad patrimonial y a que posee atractivos naturales y artificiales. Sin embargo, no ha tenido fuerza en el manejo de una adecuada publicidad.

En el caso de Puebla, no posee aeropuerto, pero ha mantenido un destacado crecimiento en el turismo 
porque ha utilizado estrategias útiles para atraer turistas, mientras que la ciudad de Cuenca posee un aeropuerto de vuelos locales y, no ha tenido un resultado favorable con la llegada de turistas. La ciudad de mayor ventaja por poseer un aeropuerto con llegadas internacionales ha sido Cusco, dicha situación ha permitido que se coloque en la delantera del turismo en Latinoamérica, pero también se debe a que no ha desaprovechado las oportunidades de mejora y promoción.

Además, los metabuscadores como Booking y Expedia muestran las ofertas de atractivos de cada ciudad, pero adicional a eso la planta de alojamiento, tanto con las ofertas de alimentación y entretenimiento. En el caso de Puebla y Cusco, tiene una gama variada de opciones. Por tanto se considera que es importante acoger el ejemplo de desarrollo turístico de otras ciudades y buscar nuevas estrategias de gestión, además de convenios que podrían beneficiar en la potencialización del turismo y establecer regulaciones que ayuden a favorecer al sector turístico en general

\section{Referencias.}

Ajpis, C. (31 de junio de 2015). Los 10 criterios para declarar un sitio como patrimonio de la humanidad. AM de Querétaro. Recuperado de https://bit.ly/3c9JiYf

Boullón, R. (2006). Planificación del espacio turístico. Argentina: Trillas. Recuperado de https://bit.ly/2zBqx2x

Booking. (s.f). Busca ofertas en hoteles, casas. Obtenido de Booking:

https://www.booking.com/index.es.html

Cantorán, J. (15 de enero de 2018). ¿Llegaron a Puebla 14 millones de turistas en 2017? La Dobe. Recuperado de https://bit.ly/2M6FvAg

Cañizares Ruíz, M. (2013). Sostenibilidad y turismo: de la documentación interna a la planificación en España (Horizonte 2020). Boletín de la Asociación de Geógrafos Españoles (61), 67-92.
DataTur. (2017). Encuesta de Viajeros Internacionales. Obtenido de Instituto Nacional de Estadística y Geografía: https://bit.ly/2XFjQoh

DataTur. (2017). Puebla. Obtenido de DataTur: https://bit.ly/3hozWqz

Dejtiar, F. (25 de octubre de 2017). Guía de arquitectura en Cuenca: 10 sitios que todo arquitecto debe conocer. Plataforma arquitectura. Recuperado de https://bit.ly/2XxgRoW

Despegar. (s.f.). Viajes baratos y experiencias únicas de viajes. Obtenido de Despegar:

https://www.despegar.com.ec/

El Comercio. (24 de julio de 2016). Cuenca tiene el perfil de su turista. Recuperado de https://bit.ly/3ermDrP

El Economista. (10 de febrero de 2018). Booking.com y Expedia, principales canales de distribución de reservas hoteleras en España. Recuperado de https://bit.ly/2X9q2pk

ENPERÚ. (2015). Información de Cusco. Obtenido de ENPERÚ: https://bit.ly/2Acq86A

Expedia. (s.f.). Buscar hospedaje. Obtenido de Expedia: https://bit.ly/2ZIqdtr

Fundación Municipal Turismo para Cuenca. (2018). Rutas turísticas dentro de la ciudad de Cuenca. Recuperado de

http://cuencaecuador.com.ec/rutas-recomendadas

Get your Guide. (2018). Cuzco, Tours de arquitectura. Recuperado de https://bit.ly/2X7YsZH

Lickorish, L.J \& Jenkins, C (1997) Una introducción al turismo. Madrid, España: Síntesis, S, A 
Molina Collado, A., Consuegra Navarro, D. M., Talaya, Á. E., \& Díaz Sánchez, E. (2007). Segmentación de la demanda turística: un análisis aplicado a un destino de turismo cultural. Revista de Análisis Turístico, 36-48.

Monfort, V. (2000). La política turística: Una aproximación. Cuadernos de Turismo (6). 7-27. Recuperado de https://bit.ly/2YwkRPU

Morantes, V. (2015). ¿Cómo el precio del dólar afecta la comunidad colombiana? Recuperado de https://bit.ly/3gwZkOY

Moya Torres, A. (1994). El auge de la cascarilla y su contexto histórico. En L. Cascarilla, Auge y crisis de la cascarilla en la Audiencia de Quito, siglo XVIII (pp. 15-28). Quito, Ecuador: FLACSO. Obtenido de https://bit.ly/3et7TIK

Municipalidad del Cusco. (2015). Plan maestro del Centro Histórico del Cusco. Obtenido de Municipalidad del Cusco: https://bit.ly/3gqszmG

Municipio de Cuenca. (2016). Plan de ordenamiento territorial. Obtenido de Municipio de Cuenca: https://bit.ly/3gu2qUc

OMT (2005). Indicadores de desarrollo sostenible para los destinos turísticos. WTO Library. Recuperado de https://n9.cl/m9lj

PromPerú. (enero, 2017). ¿Qué hacer en Perú? Recuperado de https://www.cuscoperu.com/es/que-hacer

Perú.com. (2017). Cusco recibió más de 3 millones de turistas en el 2016. Recuperado de https://bit.ly/3cfx2Wc

Rengifo, J. (2017). Los seis ríos de Puebla principales. Recuperado de https://www.lifeder.com/rios-de-puebla/
Rozas, P., \& Sánchez, R. (2004). Desarrollo de infraestructura y desarrollo económico: revisión conceptual. CEPAL. Serie 75. Recursos naturales e infraestructura. Santiago. Recuperado de https://n9.cl/4z8p

RPP Noticias. (18 de agosto 2013). Cusco se ubica en segundo lugar en campaña ciudades Maravilla del Mundo. Recuperado de https://bit.ly/2XCGmoY

Serrano, A. L., Freire, S., Sanmartín, I., Espinoza, F., Farfán, K., Andrade, S., \& Villafuerte, E. (2018). Boletín de indicadores turísticos del cantón Cuenca. Cuenca: Universidad de Cuenca.

UNWTo (2017). El turismo: un fenómeno económico y social. Recuperado de https://bit.ly/3dofVrx

Vélez, M. A. (2015). Las relaciones Colombia, Venezuela, Ecuador y Perú en el contexto de los procesos de integracción socioeconómica y sociopolítica en América Latina (2002 - 2011). Revista Fundación Universitaria Luis Amigó, 149-168.

Vizcarra, M. (03 de noviembre de 2014). Así son los turistas que visitan Puebla. Unión Puebla. Recuperado de https://bit.ly/2ZJsJzu 